\title{
Evaluación ultrasonográfica de la función endotelial en niños y adultos chilenos
}

\author{
Alfredo M G ermain $A^{1}$, Verónica Irribarra $P^{2}$, \\ G loria Valdés $\mathrm{S}^{3}$, Mary Carmen Romanik Fla, \\ Federico Leighton $\mathrm{P}^{4}$, Francisco Mardones $\mathrm{S}^{5}$ y \\ Ada Cuevas $\mathrm{M}^{2}$.

\section{Ultrasound assessment of endothelial function in Chilean children and adults}

Background: Endothelial dysfunction is an important pathogenetic mechanism in the development of atherosclerosis. Aim: To evaluate endothelial function in Chilean children and adult subjects and to provide normal values of flow mediated dilatation (FMD) in the Chilean population. Subjects and Methods: Flow mediated dilation of the brachial artery was measured by high resolution ultrasonography in healthy children $(n=32)$ and adults $(n=69)$ of both gender, in a group of 8 healthy women during 4 periods of pregnancy and late postpartum, and in 22 men and women with a history of stroke or coronary heart disease. Results: FMD in boys and girls was $9.9 \pm 3.6$ and $10.0 \pm 4.2 \%$ respectively (NS). The figures for young women and young men were $11.3 \pm 3.8$ and $8.6 \pm 3.9$, respectively ( $p=0.02$ ); for postmenopausal women and older men, $5.5 \pm 6.6$ and $7.6 \pm 6.7$ respectively (NS). During normal pregnancy and postpartum there were no significant changes in FMD. Patients with cardiovascular disease had a FMD of $0.3 \pm 5.2 \%$, ( $p<0.001$, with other groups). Conclusions: The present study provides values of FMD in healthy Chilean subjects of different ages, and in patients with coronary heart disease (Rev Méd Chile 2004; 132: 437-44).

(Key Words: Atherosclerosis; Endothelium; Vascular ultrasonography)

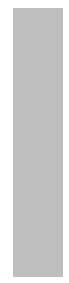

Recibido el 6 de marzo, 2003. Aceptado en versión corregida el 21 de enero, 2004.

Financiamiento: Proyecto Fondecyt \#1020705, Programa «Bases Moleculares de las Enfermedades Crónicas» PUC Proyecto Investigación y Desarrollo Laboratorios Gynopharm, Grupo Recalcine.

Departamento de Obstetricia y Ginecología1, Nutrición, Diabetes y Metabolismo², Nefrología ${ }^{3}$ y Salud Pública ${ }^{5}$, Facultad de Medicina, Departamento de Biología Celular y Molecular, Facultad de Ciencias Biológicas ${ }^{4}$, Pontificia Universidad Católica de Chile. aEnfermera Matrona

Correspondencia a: Alfredo M Germain. Marcoleta 340, Santiago, Chile. Fono: (562) 6863034. Fax: (562) 2071069.

E mail: agermain@med.puc.cl 
$\mathrm{E}^{\mathrm{n}}$ los últimos 15 años se ha evidenciado el rol crucial del endotelio en la homeostasis vascular, cuya localización estratégica, entre la sangre y el músculo liso, le permite contribuir a regular el tono vasomotor y las funciones de las células sanguíneas ${ }^{1,2}$. Por otro lado, el endotelio es el blanco primario de los factores de riesgo cardiovascular, y se ha demostrado que las alteraciones patológicas asociadas a la aterosclerosis y a sus factores de riesgo (hipercolesterolemia, diabetes mellitus, hipertensión arterial y tabaquismo), afectan la síntesis de las sustancias vasoactivas derivadas del endotelio. Esto condiciona las modificaciones funcionales y estructurales responsables del desarrollo y progresión de la enfermedad aterosclerótica ${ }^{3,4}$.

Actualmente es posible evaluar la función endotelial a través de la medición de la dilatación vascular dependiente del endotelio, mediante ultrasonografía de alta resolución de la arteria braquial, una metodología simple, reproducible y no invasiva. A través de este método, se ha determinado la función endotelial en distintos grupos poblacionales y evaluado el impacto de intervenciones farmacológicas y no farmacológicas $^{6,7}$. En nuestro país, no se cuenta con datos de función endotelial en individuos sanos, en diferentes momentos de la vida. El objetivo de este trabajo es determinar mediante ultrasonografía de arteria braquial la función endotelial en niños y adultos chilenos sanos, en embarazadas normales y en pacientes portadores de patología cardiovascular. La información obtenida será relevante para establecer patrones de referencia y el diseño de futuros estudios de intervención en la población chilena.

\section{Materiales y MÉTOdos}

El estudio se realizó entre enero de 1999 y diciembre de 2001 en la Unidad de Evaluación de la Función Endotelial de la Facultad de Medicina de la Pontificia Universidad Católica.

Población. Las características generales de los individuos sanos incluidos en el estudio se describen en la Tabla 1.

Niños: 32 niños de ambos sexos (19 niñas y 13 varones), sanos, de peso normal, provenientes de consultorios de las comunas de Puente Alto y La Florida, cuya edad fue 9,9 $\pm 0,2$ (promedio \pm error estándar) [rango: 9 a 10] años. De acuerdo a los criterios de inclusión, todos ellos tenían un índice de masa corporal (IMC) entre el percentil 25 y 75 para su edad, de $17,4 \pm 1,7 \mathrm{~kg} / \mathrm{m}^{2}$ para las mujeres y $17,1 \pm 2,0 \mathrm{~kg} / \mathrm{m}^{2}$ para los varones. Se consideró presión arterial normal a valores $<120 / 80 \mathrm{mmHg}$

Tabla 1. Características generales de los individuos

\begin{tabular}{|lcccccc|}
\hline & \multicolumn{2}{c}{ Niños } & \multicolumn{2}{c}{ Adultos jóvenes } & \multicolumn{2}{c|}{ Adultos mayores } \\
& Hombres & Mujeres & Hombres & Mujeres & Hombres & Mujeres \\
& 13 & 19 & 22 & 24 & 13 & 10 \\
$\mathrm{n}$ & $10,0 \pm 0,0$ & $9,9 \pm 0,2$ & $22,4+2,6$ & $33,3 \pm 4,1$ & $63,3 \pm 3,5$ & $64,0 \pm 4,3$ \\
Edad (años) & $17,1 \pm 2,0$ & $17,4+1,7$ & $23,1 \pm 2,0$ & $22,8 \pm 3,0$ & $26,6 \pm 3,8$ & $26,9 \pm 3,0$ \\
IMC (k/m²) & $102,3 \pm 9,0$ & $99,8 \pm 10,8$ & $118,9 \pm 13,1$ & $107,1 \pm 10,4^{*}$ & $139,1 \pm 15,8$ & $137,5 \pm 17,3$ \\
PAS (mmHg) & $55,8 \pm 7,8$ & $54,6 \pm 6,1$ & $63,1 \pm 6,9$ & $60,5 \pm 8,0$ & $78,3 \pm 5,2$ & $71,3 \pm 11,0$ \\
PAD (mmHg) & $138,4 \pm 24,4$ & $157,7 \pm 25,2$ & $175,1 \pm 26,9$ & $162,5 \pm 22,9$ & $200,8 \pm 24,4$ & $211,5 \pm 36,1$ \\
CT (mg/dl) & $77,8 \pm 21,6$ & $95,2 \pm 24,6$ & $110,2 \pm 21,5$ & $101,0 \pm 22,9$ & $130,0 \pm 23,9$ & $135,2 \pm 27,3$ \\
C LDL (mg/dl) & $43,1 \pm 6,9$ & $42,4+11,9$ & $49,7 \pm 9,5$ & $45,0 \pm 10,2$ & $46,2 \pm 11,4$ & $55,7 \pm 15,4$ \\
C HDL (mg/dl) & $87,8 \pm 34,0$ & $101,0 \pm 43,1$ & $73,8 \pm 38,4$ & $75,0 \pm 28,2$ & $123,8 \pm 70,0$ & $103,0 \pm 42,9$ \\
TG (mg/dl) & $81,0 \pm 7,3$ & $83,3 \pm 7,7$ & $71,7 \pm 6,5$ & $90,2 \pm 7,4$ & $99,5 \pm 8,7$ & $92,5 \pm 10,6$ \\
Glicemia (mg/dl) & & & & & \\
\hline
\end{tabular}

$\mathrm{IMC}=$ Indice de masa corporal, $\mathrm{PAS}=$ Presión arterial sistólica, $\mathrm{PAD}=$ Presión arterial diastólica, $\mathrm{CT}=$ colesterol total, $\mathrm{C} \mathrm{LDL}=$ colesterol LDL, C HDL= colesterol HDL, TG = triglicéridos. * $p=0,002$, respecto a hombres jóvenes. Promedio \pm DS. 
de acuerdo a «Hipertensión Arterial Normas Técnicas»), MINSAL (Eds. MC Escobar, G Valdés; Programa Salud del Adulto, 1995).

Adultos jóvenes: 46 adultos jóvenes (22 hombres y 24 mujeres) sanos. Todas las mujeres habían tenido al menos un embarazo y parto normal, presentaban ciclos menstruales regulares, no consumían anticonceptivos orales y se encontraban en día 10 de la fase lútea del ciclo menstrual (10 días después de una ovulación confirmada ecográficamente). Las mujeres tenían 33,3 $\pm 4,1$ (rango: 25 a 39) años de edad, con IMC $22,8 \pm 3,0(16,5$ a 28,9$) \mathrm{kg} / \mathrm{m}^{2}$. Los hombres jóvenes tenían 22,4 $\pm 2,6$ (rango: 19 a 28) años, un IMC $23,1 \pm 2,0$ (rango: 19,4 a 27,1 ) $\mathrm{kg} / \mathrm{m}^{2}$ y todos presentaban presión arterial normal para su edad.

Adultos mayores: 23 individuos de ambos sexos (10 mujeres y 13 hombres) provenientes del Centro del Adulto Mayor de la comuna de Providencia. Las 10 mujeres eran postmenopáusicas y no recibían terapia hormonal de reemplazo, tenían 64,0 04,3 (rango: 58 a 73) años de edad y un IMC entre 18,8 y $34 \mathrm{~kg} / \mathrm{m}^{2}$, los 13 hombres tenían 63,3 $\pm 3,5$ (rango: 55 a 70) años de edad y un IMC entre 18,8 y $33,9 \mathrm{~kg} / \mathrm{m}^{2}$.

Se excluyó a sujetos con obesidad severa (IMC $>35 \mathrm{~kg} / \mathrm{m}^{2}$ ), hábito tabáquico, dislipidemia (colesterol total $>240 \mathrm{mg} \%$ o triglicéridos $>250 \mathrm{mg} \%$ ), hipertensión arterial (presión arterial >140/90 $\mathrm{mmHg}$ o en tratamiento antihipertensivo), glicemia de ayuno $>126 \mathrm{mg} \%$ o antecedente de diabetes mellitus, enfermedad coronaria u otra enfermedad cardiovascular, insuficiencia renal o hepática, hipotroidismo no controlado, cáncer y otras enfermedades sistémicas. También se excluyeron individuos que consumían medicamentos o suplementos que pudiesen modificar la función endotelial, como aspirina, hipolipemiantes, inhibidores de la enzima convertidora de angiotensina, antagonistas del receptor de angiotensina II, antagonistas del calcio, sildenafil, L-arginina y antioxidantes.

Embarazadas: 8 mujeres de 29,9 $\pm 3,8$ (rango: 27 a 33) años, con historia reproductiva normal, fueron evaluadas en forma longitudinal en cuatro períodos del embarazo y postparto tardío (6 semanas). Todas las pacientes tuvieron un embarazo fisiológico con parto de término $(38,9 \pm 0,9$ sem) y recién nacidos normales $(3.416 \pm 438 \mathrm{~g}$, Apgar 9-9).
Pacientes con patología cardiovascular: 22 pacientes (14 hombres y 7 mujeres), promedio de edad de 58,3 $\pm 12,5$ (rango 29 a 72 años), portadores de enfermedad coronaria o cerebrovascular, comprobada (eventos clínicos previos y electrocardiograma/cintigrafía de esfuerzo positivo, tomografía computada cerebral, respectivamente). El $44 \%$ de ellos se encontraba en tratamiento antihipertensivo con antagonistas del calcio, diuréticos o antagonistas del receptor de angiotensina, un sujeto recibía atorvastatina y 2 mujeres estrógenos. Ninguno presentaba diabetes mellitus.

Metodología. Todos los sujetos permanecieron en ayunas por $12 \mathrm{~h}$ antes del examen, ocasión en que además se realizó una anamnesis, se determinó el peso y la talla, y presión arterial mediante un monitor de presión arterial Dynamap (Johnson \& Johnson Inc. USA), con el sujeto en reposo en decúbito dorsal por $10 \mathrm{~min}$. Posteriormente, se midió la dilatación vascular dependiente de endotelio por ultrasonografía de la arteria braquial y se les extrajo una muestra de $5 \mathrm{ml}$ de sangre venosa para la determinación de lípidos plasmáticos y glicemia. El protocolo de este estudio fue aprobado por el Comité de Ética de la Facultad de Medicina de la Pontificia Universidad Católica de Chile. Todos los sujetos adultos entregaron su consentimiento informado y en el caso de los niños, éste fue dado por, al menos, uno de sus padres.

\section{Determinaciones de laboratorio.}

Determinaciones plasmáticas: Se tomaron muestras de sangre en tubos con EDTA. El plasma fue separado por centrifugación a $2.000 \mathrm{x}$ g, durante $3 \mathrm{~min}$, a $4^{\circ} \mathrm{C}$. El colesterol total y triglicéridos fueron determinados por métodos enzimáticos (Boehringer Mannheim, Mannheim, Alemania). El colesterol-LDL fue calculado por la fórmula de Friedewald ${ }^{8}$ y el colesterol-HDL, por precipitación con cloruro de magnesio ${ }^{9}$. La glicemia fue determinada por método de glucosa oxidasa ${ }^{10}$.

Ultrasonografía vascular de alta resolución en la arteria braquial: Se utilizó el método no invasivo de ultrasonografía vascular de alta resolución ${ }^{5,10-14}$. El examen se realizó en ayunas, con instrucciones de no fumar en las $12 \mathrm{~h}$ previas y evitar ingesta de comidas grasas en la noche anterior. Además, los pacientes con patología cardiovascular, suspendieron, al menos $12 \mathrm{~h}$ pre- 


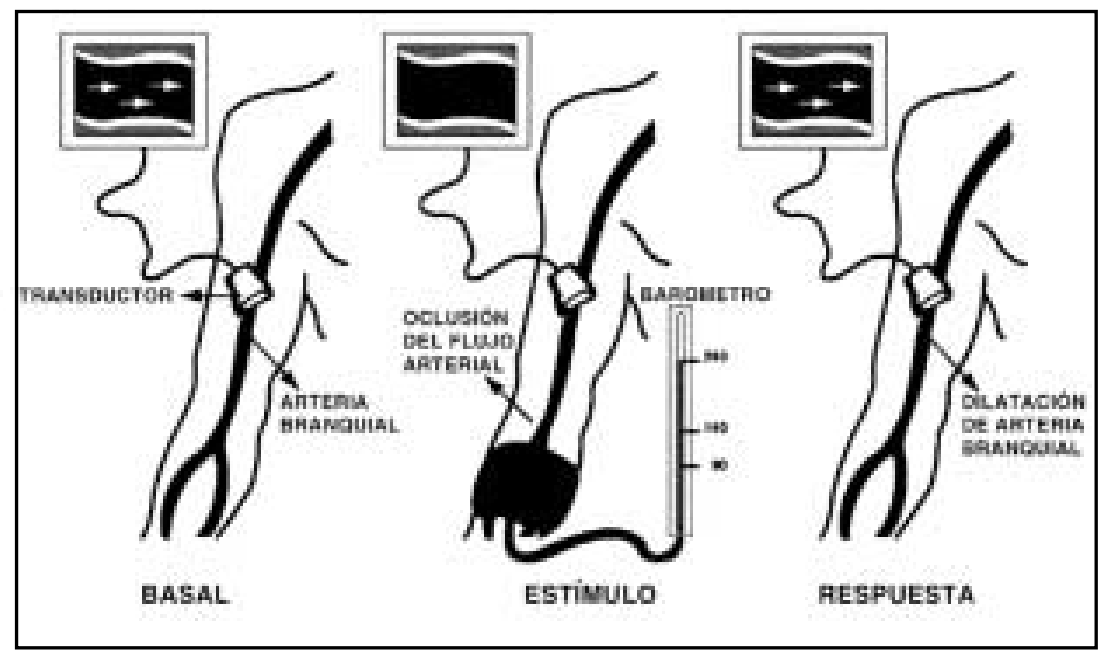

FIgURA 1. Técnica de evaluación de la función endotelial: Basal= El transductor localiza la arteria braquial y se mide su diámetro. Estímulo= Obstrucción al flujo arterial por aplicación de una presión de $200 \mathrm{mmHg}$ por 5 min a través del manguito de presión. Respuesta $=$ Registro del cambio de diámetro arterial 1 min después de liberar la presión.

vias al examen, los fármacos que conocidamente modifican la función endotelial. Las mediciones se realizaron en una habitación con temperatura controlada a $22^{\circ} \mathrm{C}$, entre las 8:00 y 10:00 AM. La imagen de la arteria braquial se visualizó utilizando un transductor lineal con una frecuencia de 10,5 MHz (Medison, GAIA MT, Seul, República de Corea), captando una vista longitudinal de la arteria en un segmento de aproximadamente 5 $\mathrm{cm}, 3 \mathrm{a} 7 \mathrm{~cm}$ sobre el pliegue antecubital, en el brazo no dominante del sujeto, que se mantuvo inmovilizado en extensión. La ganancia del equipo se ajustó para obtener óptima delineación de la pared arterial (interfaz). Las mediciones se coordinaron al final de diástole. Luego de determinar el diámetro basal de la arteria, se colocó un manguito de presión sobre la muñeca y se aplicó una presión de $200 \mathrm{mmHg}$ por 5 min para luego soltar rápidamente la oclusión (Figura 1). Al minuto postoclusión, se realizaron tres mediciones del diámetro arterial, calculando la dilatación en respuesta a la hiperemia reactiva o dilatación mediada por endotelio (DME), como el porcentaje de cambio en el diámetro arterial con respecto al diámetro basal (promedio de tres mediciones). Para evaluar la capacidad de respuesta del músculo liso vascular a un estímulo exógeno, en los adultos, 15 min después se realizó una evaluación de vasodilatación muscular directa inducida por nitroglicerina (NTG) (0,3 mg por vía sublingual), con excepción de los niños y las mujeres embarazadas; realizándose tres mediciones del diámetro de la arteria 3 min después. Las mediciones del diámetro arterial se obtienen a partir del análisis del registro digital de la imagen en el mismo lugar (Figura 2). Se consideró disfunción endotelial una $\mathrm{DME}<4,5 \%{ }^{15}$. Para evaluar la variación del examen, en otro grupo de 15 sujetos sanos, se realizó la determinación de función endotelial en forma repetida con intervalos de 2 a 7 días. El coeficiente de variación interensayo fue de $4,5 \%$.

Análisis estadístico. Todas las variables fueron expresadas como promedio \pm error estándar. Los cálculos de valor $\mathrm{p}$ se consideraron los valores absolutos. Para comparar grupos de pacientes se utilizó Análisis de Varianza (ANOVA), con programa Sigma Stat 2.03 para Windows (SPSS Inc., Chicago Illinois). Para la determinación de comelaciones entre las variables estudiadas se utilizó análisis de regresión múltiple, y se consideró estadísticamente significativo un valor de $\mathrm{p}$ menor a 0,05 .

\section{Resultados}

Dilatación mediada por endotelio (Figura 3).

Niños: La DME fue de $10 \pm 4,2 \%$; y $9,9 \pm 3,6 \%$ en niñas y niños respectivamente (NS). El 12,5\% de los niños (4/32) evidenciaron una disfunción endotelial.

Adultos jóvenes: Las mujeres presentaron una mayor dilatación mediada por flujo en comparación a los hombres jóvenes $(11,3 \pm 3,7 \%$ versus 
$8,6 \pm 3,9 \% ; p=0,02)$. Ninguna de las mujeres presentó una DME inferior a 4,5\%, en cambio $13,6 \%$ (3/ 22) de los hombres evidenciaron una disfunción endotelial.

Adultos mayores: Las mujeres presentaron una menor DME en comparación a las niñas y mujeres jóvenes $(5,5 \pm 6,6 \%$ versus $10 \pm 4,2 \%$ y $11,3 \pm 3,7 \%$ respectivamente; $\mathrm{p}<0,05$ y $\mathrm{p}<0,04$ respectivamente). Los hombres mayores presentaron una DME de 7,6 $6,6 \%$ (NS) sin diferencias significativas con los jóvenes ni con los niños. No se observaron diferencias por sexo en este grupo
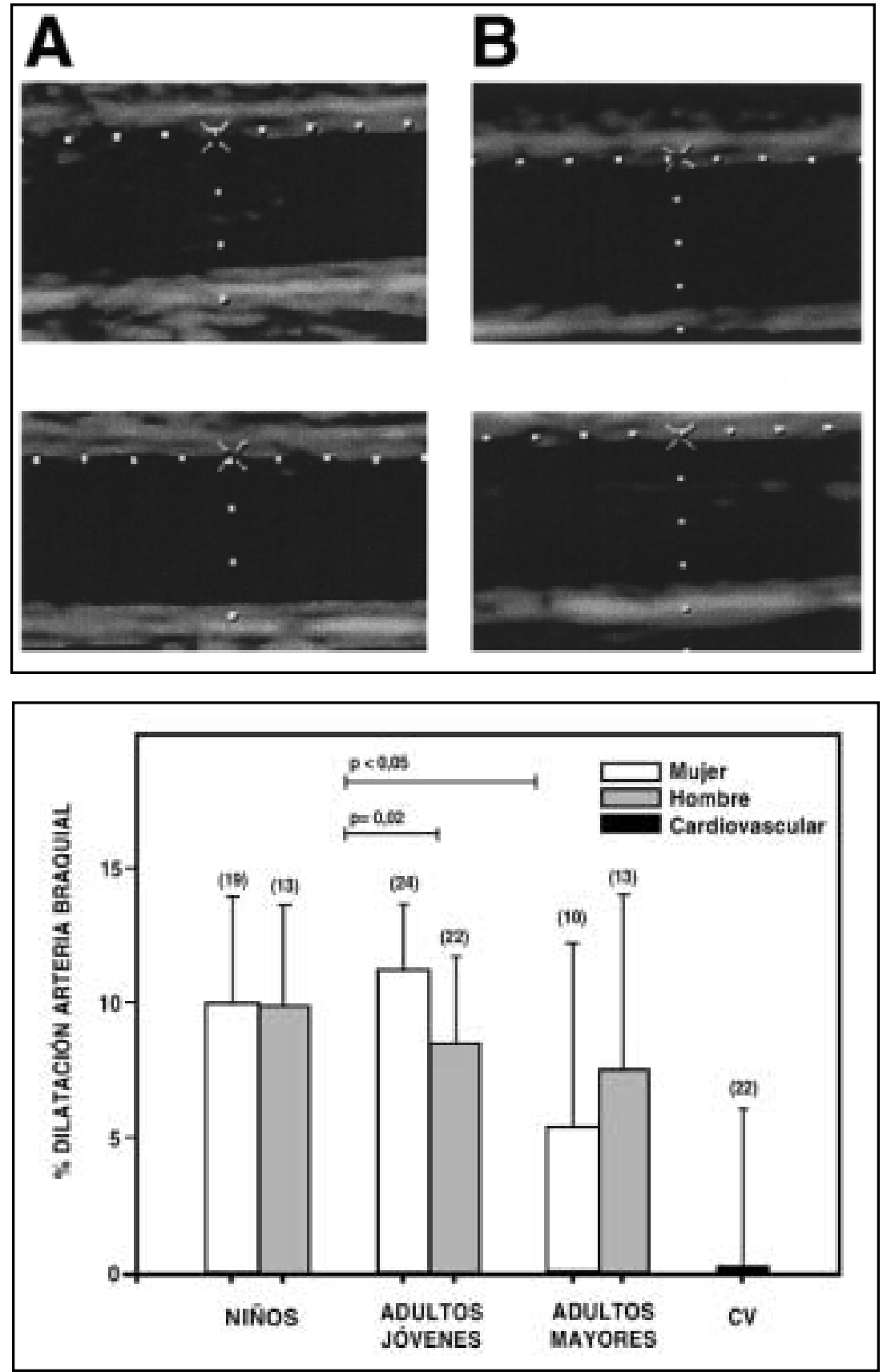

FiguRA 2. Imagen digital de la arteria braquial en un sujeto sano y en un paciente con enfermedad coronaria: $\mathrm{A}=$ arteria braquial con diámetro inicial de $0,27 \mathrm{~mm}$ y postoclusión de $0,31 \mathrm{~mm}$ con una dilatación de $17,0 \% . \quad B=$ arteria braquial con diámetro inicial de 0,28 $\mathrm{mm}$ que postoclusión disminuye su diámetro a 0,27 mm con una variación de $2,2 \%$.

Figura 3. Función endotelial en arteria braquial de sujetos controles. Porcentaje de dilatación de arteria braquial 1 min postoclusión en mujeres y hombres de diferentes grupos etáreos. Promedio \pm DS, entre paréntesis número de sujetos de cada grupo. 
etáreo. Veinte por ciento de las mujeres mayores $(2 / 10)$ y $38 \%$ de los hombres $(5 / 13)$ presentaron una disfunción endotelial.

Embarazadas: Entre la $6^{\underline{a}}$ y $7 \stackrel{a}{a}+6$ semanas de embarazo se observó una dilatación de $8,6 \pm 4,2 \%$, la que tendió a aumentar a $12,0 \pm 4,3 \%$ entre la $14^{\text {a }}$ y $15^{\underline{a}}+6$ semanas, a $11,1 \pm 6,4 \%$ entre la $24^{\underline{a}}$ y $27 \underline{a}+6$ semanas y a $13,5 \pm 6,7 \%$ entre la $32^{\underline{a}}$ y $38^{\underline{a}}+6$ semanas (NS). A las 6 semanas de postparto la DME fue $9,8 \%$, sin diferencias significativas con las etapas de embarazo evaluadas (NS).

Pacientes con patología cardio o cerebrovascu-

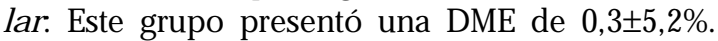
Sólo 3 sujetos presentaron una DME superior a $4,5 \%$, y el resto (19/22, 86\% de ellos) evidenció disfunción endotelial.

Correlación entre DME y lípidos plasmáticos, glicemia y presión arterial: No se observó una correlación significativa entre la DME y los lípidos plasmáticos, glicemia y presión arterial en ninguno de los grupos estudiados $(r=0,28, p>0,05)$.

Dilatación vascular con nitroglicerina (Tabla 2). Dada la posibilidad de un descenso de la presión arterial, en los niños y embarazadas no se realizó esta intervención. En los adultos se observó que la dilatación vascular inducida por nitroglicerina fue significativamente mayor en las mujeres en comparación a los hombres. Además las mujeres jóvenes presentaron una mayor dilatación con NTG en comparación a los hombres jóvenes $(\mathrm{p}=0,001)$. Los sujetos con patología cardiovascular no presentaron diferencias significativas de dilatación inducida por NTG en comparación a los sujetos sanos. El examen fue bien tolerado, observándose cefalea transitoria y eritema facial secundarios a la administración de nitroglicerina en 10\% de mujeres y $5 \%$ de hombres adultos.

\section{DisCUSIÓN}

Los resultados de nuestro estudio revelan valores de DME comparables a otras poblaciones reportadas en la literatura, tanto en niños como en adultos $11,16-18$. Destaca la mayor DME observada en mujeres de edad fértil en comparación al grupo de mujeres postmenopáusicas y a hombres de cualquier edad, posiblemente debido al efecto beneficioso de los estrógenos sobre la función endotelial ${ }^{19-21}$. Por otra parte, nuestros resultados evidencian una disminución de la DME con la edad, tanto en hombres como en mujeres, lo cual también es concordante con lo reportado en otros grupos poblacionales ${ }^{22-24}$.

Otro resultado interesante fue la detección de sujetos que presentaron una DME sugerente de disfunción endotelial en todos los grupos etáreos, pero principalmente en los adultos mayores. Esto llama la atención dado que se seleccionaron individuos que no presentaron factores causantes conocidos de disfunción del endotelio. Este hallazgo, unido a la falta de asociación entre DME y lípidos plasmáticos, glicemia y presión arterial, sugiere que probablemente existen variables aún no conocidas que alteran la dilatación mediada por endotelio y que podrían relacionarse con la dieta, como ha sido sugerido por nuestro grupo ${ }^{6} 0$ incluso con factores prenatales ${ }^{25,26}$.

En las embarazadas hubo una tendencia al aumento de la DME entre el inicio embarazo y el segundo y tercer trimestre. Esta tendencia se revierte en las mediciones postparto en que retorna a cifras de $9 \%$. Si bien las diferencias no aparecen como estadísticamente significativas, dado lo pequeño de la serie estudiada, los cambios pueden asociarse a la adaptación vascular al embarazo, como ha sido reportado 27,28 .

Tabla 2. D ilatación mediada por endotelio e inducida por nitroglicerina

\begin{tabular}{|c|c|c|c|c|c|}
\hline & \multicolumn{2}{|c|}{ Adultos jóvenes } & \multicolumn{2}{|c|}{ Adultos mayores } & \multirow[t]{2}{*}{ Cardiovascular } \\
\hline & Hombres & Mujeres & Hombres & Mujeres & \\
\hline DME (\%) & $8,6 \pm 3,9$ & $11,3 \pm 3,7$ & $7,60 \pm 6,3$ & $5,5 \pm 6,6$ & $0,3 \pm 5,2$ \\
\hline NTG (\%) & $22,0 \pm 6,0$ & $30,8 \pm 7,9 *$ & $14,6 \pm 6,8$ & $19,9 \pm 12,0$ & $13,8 \pm 3,2$ \\
\hline
\end{tabular}

$\mathrm{DME}=$ Dilatación mediada por endotelio. $\mathrm{NTG}=$ Dilatación inducida por nitroglicerina. ${ }^{*} \mathrm{p}=0,001$ respecto a hombres jóvenes. Promedio \pm DS. 
Finalmente, detectamos que todos los sujetos portadores de patología cardiovascular presentaban una severa disfunción endotelial, a pesar de que ellos recibían fármacos que mejoran la función endotelial (estatinas, antagonistas del calcio o inhibidores de enzima convertidora). Existe amplia evidencia respecto a la relevancia de la disfunción endotelial en el riesgo cardiovascular, mostrando la utilidad de este método no invasivo como herramienta para evaluar la integridad de funcional de la pared vascular, entregando información diagnóstica y pronóstica. Elderle y $\operatorname{cols}^{29}$ estudiaron a 122 pacientes con sospecha de enfermedad coronaria que fueron sometidos a catetenización ventricular izquierda y evaluación de la función endotelial en arteria braquial. Valores de DME menores a 4,5\% mostraron una sensibilidad, especificidad y valor predictivo de $71 \%$, $81 \%$ y $95 \%$, respectivamente para el diagnóstico de enfermedad coronaria. En el análisis que hace Schroeder y cols, de este tipo de pacientes, el mismo punto de corte para DME alterada, resultó casi tan sensible en el diagnóstico de enfermedad comnaria como el electrocardiograma de esfuerzo y más específica para el diagnóstico que angina pectoris y electrocardiograma de esfuerzo ${ }^{15}$.

La disminución en la respuesta a nitroglicerina con la edad -atribuible a un deterioro progresivo

\section{REFERENCIAS}

1. De Caterina R. Endothelial dysfunctions: Common denominators in vascular disease. Curr Opin Lipidol 2000; 11: 9-23.

2. Cines DB, PoLak ES, Buck CK, Loscaizo JL, ZiMMERMAN GA, McEver RP ET aL. Endothelial cells in physiology and in the pathophysiology of vascular disorders. Blood 1998; 91: 3527-61.

3. QuYYumi AA. Endothelial function in health and disease: new insights into the genesis of cardiovascular disease. Am J Med 1998; 105: 32S-39S.

4. Kinlay S, Libby P, Ganz P. Endothelial function and coronary artery disease. Curr Opin Lipidol 2001; 12: 383-9.

5. Irribarra V, Germain AM, Cuevas AM, Faúndez L, VALDÉs G. Disfunción endotelial como alteración primaria en las patologías vasculares. Rev Méd Chil 2000; 128: 659-70.

6. Cuevas AM, Guasch V, CastiLo O, Irribarra V, Mizón C, San Martín A et al. A high fat diet induces and de la función del músculo liso- también se ha reportado previamente ${ }^{30}$. Adicionalmente detectamos que las mujeres jóvenes presentan una mejor respuesta a NTG en comparación a los hombres jóvenes, lo cual nos hace plantear un efecto beneficioso de los estrógenos sobre el músculo liso vascular. Concordante con esta hipótesis, no se detectó una diferencia significativa en la respuesta vascular a NTG entre las mujeres (postmenopáusicas) y los hombres mayores.

Nuestros resultados de función endotelial determinada por la medición de DME en individuos chilenos estrictamente seleccionados según criterios nutricionales, metabólicos y hormonales, son comparables a los datos de otros grupos poblacionales y proveen un adecuado patrón de referencia para futuros estudios. Además, las diferencias detectadas de DME en los distintos grupos, atribuibles al sexo, edad o estatus hormonal, son concordantes con lo reportado previamente. Esto, sumado al coeficiente de variabilidad de nuestro grupo para esta metodología $(4,5 \%)$, permiten establecer un alto grado de calidad y reproducibilidad en una medición que permite, precozmente, detectar el compromiso vascular en una etapa en la que las intervenciones preventivas pueden tener una mayor efectividad que frente a la enfermedad ateromatosa establecida.

red wine counteracts endothelial dysfunction in human volunteers. Lipids 2000; 35: 143-8.

7. Cuevas aM, Irribarra VL, CastiLo OA, Yáñez MD, GERMAIN AM. Isolated soy protein improves endothelial function in postmenopausal hypercholesterolemic women. European Journal Clinical Nutrition 2003; 57: 889-94.

8 FriedewaLd WT, LevY IR, FREDERICKSON DS. Estimation of the concentration of low density lipoprotein cholesterol in plasma, without use of preparative ultracentrifuge. Clin Chem 1972; 18: 499-502.

9. Schwandt P, Weisweiler P. Determination of cholesterol and phospholipid concentrations in high density lipoproteins by heparin-manganese chloride precipitation. Clin Chim Acta 1979; 95: 395-9.

10. BARTON RR. A specific method for quantitative determination of glucose. Anal Biochem 1966; 14: 258-60.

11. KeLM M. Flow mediated dilatation in human circulation: diagnostic and therapeutic aspects. Am J Physiol: Heart Circ Physiol 2002; 282: H1-H5. 
12. Celermajer DS, Sorensen KE, Gooch VM, SpiegelhalTER DJ, Miluer OI, Sulivan ID et aL. Non invasive detection of endothelial dysfunction in children and adults at risk of atherosclerosis. Lancet 1992; 340: 1111-5.

13. Playford DA, Watts GF. Special article: Non invasive measurement of endothelial function. Clin Exp Pharmacol Physiol 1998; 28: 640-3.

14. Vogel RA. Measurement of endothelial function by brachial artery flow mediated vasodilation. Am J Cardiol 2001; 88(suppl): 31E-34E.

15. Schroeder S, Enderle MD, Ossen R, Meissner C, Baumbach A, Pfohl M et al. Non invasive determination of endothelium mediated vasodilation as a screening test for coronary artery disease: pilot study to assess the predictive value in comparison with angina pectoris, exercise electrocardiography, end myocardial perfusion imaging. Am Heart J 1999; 138 (4pt 1): 731-9.

16. Correti MC, Anderson TJ, Benjamin EJ, Celermajer D, Charbonneau F, Creager MA ET al. Guidelines for the ultrasound assessment of endothelial dependent flow mediated vasodilation of the brachial artery; a report of the International Brachial Artery Reactivity Task. J Am Coll Cardiol 2002; 39: 257-65.

17. Jarvisalo MJ, Ronnemaa T, Volanen I, Kaitosaari T, KaLiO K, HaRtiala JJ ET AL. Brachial artery dilatation responses in healthy children and adolescents. Am J Physiol: Heart Circ Physiol 2002; 282: H87-H92.

18. Lieberman EH, Gerhard MD, Uehata A, Selwyn AP, Ganz P, Yeung AC ET AL. Flow induced vasodilation of the human brachial artery is impaired in patients $<40$ years of age with coronary artery disease. Am J Cardiol 1996; 78: 1210-4.

19. Sader MA, Celermajer DS. Endothelial function, vascular reactivity and gender differences in the cardiovascular system. Cardiovasc Res 2002; 53: 597-604.

20. Perregaux D, Chaudhuri A, Mohanty P, Bukhari L, WiLSON MF, Sung BH ET AL. Effect of gender differences and estrogen replacement therapy on vascular reactivity. Metabolism 1999; 48: 227-32.

21. MeNDelsohn ME, KaRas RH. The protective effects of estrogen on the cardiovascular system. NEngl J Med 1999; 340: 1801-11.
22. Ceiermajer DS, Sorensen KE, Sitegelhalter DJ, Georgakoupoulos D, Robinson J, Deanfield JE. Aging is associated with endothelial dysfunction in healthy men years before the age related decline in women. J Am Coll Cardiol 1994; 24: 471-6.

23. Jensen-Urstad K, Johansson J, Jensen-URSTAd M. Vascular function correlates with risk factors for cardiovascular disease in a healthy population of 35 years old subjects. J Intern Med 1997; 241: 507-13.

24. Lyons D, Roy S, Patel M, Benjamin N, Swift CG. Impaired nitric oxide mediated vasodilatation and total nitric oxide production in healthy old age. Clin Sci 1997; 93: 519-25.

25. Leeson CP, Whincup PH, Cook DG, Donald AE, Papacosta O, Lucas A et al. Flow mediated dilation in 9 to 11 years old children: the influence of intrauterine and childhood factors. Circulation 1997; 96: 2233-8.

26. Leeson CP, Kattenhorn M, Morley R, Lucas A, DEANFIELD JE. Impact of low birth weight and cardiovascular risk factors on endothelial function in early adult life. Circulation 2001; 6: 1264-8.

27. Dorup I SkajaA K, Sorensen KE. Normal pregnancy is associated with enhanced endothelium dependent flow-mediated vasodilation. Am J Physiol 1999; 276 (3 Pt 2): H821-H825.

28. Savvidou MD, Kametas NA, Donald AE, Nicolaides KH. Non invasive assessment of endothelial function in normal pregnancy. Ultrasound Obstet Gynecol 2000; 15: 502-7.

29. ENderle MD, Schroeder S, Ossen R, Meisner C, Baumbach A, Haering HU et al. Comparison of peripheral endothelial dysfunction and intimal media thickness in patients with suspected coronary artery disease. Heart 1998; 80: 349-54.

30. Sumulyan H, Asmar RG, Rudnicki A, London GM, SAFAR ME. Comparative effects of aging in men and women on the properties of the arterial tree. J Am Coll Cardiol 2001; 37: 1374-80.

\section{Agradecimientos}

A MEGODUC, grupo médicos gíneco-obstetras de la Universidad Católica, por las facilidades otorgadas para la utilización de equipos de ultrasonido de alta resolución, a Ana María Acosta, del Laboratorio de Nutrición, Diabetes y Metabolismo, PUC; por determinaciones de glicemia y lípidos. 